\section{RMD Open}

Rheumatic \&

Musculoskeletal Diseases

\title{
Novel protective role for MAP kinase phosphatase 2 in inflammatory arthritis
}

\author{
Juliane Schroeder, ${ }^{1}$ Kirsty Ross, ${ }^{2}$ Kathryn McIntosh, ${ }^{3}$ Shilan Jabber, ${ }^{3}$ \\ Stuart Woods, ${ }^{3}$ Jenny Crowe, ${ }^{1}$ Janet Patterson Kane, ${ }^{4}$ James Alexander, ${ }^{3}$ \\ Catherine Lawrence, ${ }^{3}$ Robin Plevin ${ }^{3}$
}

To cite: Schroeder J, Ross K, Mclntosh K, et al. Novel protective role for MAP kinase phosphatase 2 in inflammatory arthritis. RMD Open

2019;5:e000711. doi:10.1136/ rmdopen-2018-000711

- Prepublication history and additional material for this paper are available online. To view these files, please visit the journal online (http://dx.doi. org/10.1136/rmdopen-2018000711).

Received 23 April 2018 Revised 21 November 2018 Accepted 25 November 2018

Check for updates

(c) Author(s) (or their employer(s)) 2019. Re-use permitted under CC BY. Published by BMJ.

${ }^{1}$ Institute of Infection, Immunity and Inflammation, University of Glasgow, Glasgow, Scotland ${ }^{2}$ Pure and Applied Chemistry, Technology Innovation Centre, University of Strathclyde, Glasgow, Scotland

${ }^{3}$ Strathclyde Institute for Pharmacy and Biomedical Sciences, University of Strathclyde, Glasgow, Scotland ${ }^{4}$ Flagship Biosciences Inc, Massey University, Denver, Colorado, USA

Correspondence to Dr Robin Plevin; r.plevin@strath.ac.uk

\section{ABSTRACT}

Objectives We have previously shown mitogen-activated protein kinase phosphatase 2 (MKP-2) to be a key regulator of proinflammatory cytokines in macrophages. In the study presented here, we investigated the role of MKP-2 in inflammatory arthritis with a particular focus on neutrophils.

Methods To achieve this, we subjected MKP-2 deficient and wild type mice to collagen antibody induced arthritis, an innate model of arthritis, and determined disease pathology. To further our investigation, we depleted neutrophils in a prophylactic and therapeutic fashion. Last, we used chemotaxis assays to analyse the impact of MKP2 deletion on neutrophil migration.

Results $\mathrm{MKP}-2^{-/-}$mice showed a significant increase in disease pathology linked to elevated levels of proarthritic cytokines and chemokines TNF- $\alpha$, IL- 6 and MCP- 1 in comparison to wild type controls. This phenotype is prevented or abolished after administration of neutrophil depleting antibody prior or after onset of disease, respectively. While MCP-1 levels were not affected, neutrophil depletion diminished TNF- $\alpha$ and reduced $\mathrm{IL}-6$, thus linking these cytokines to neutrophils. In vivo imaging showed that MKP-2 ${ }^{-/-}$mice had an increased influx of neutrophils into affected joints, which was higher and potentially prolonged than in wild type animals. Furthermore, using chemotaxis assays we revealed that MKP-2 deficient neutrophils migrate faster towards a Leukotriene B4 gradient. This process correlated with a reduced phosphorylation of ERK in MKP-2/- neutrophils. Conclusions This is the first study to show a protective role for MKP-2 in inflammatory arthritis.

\section{INTRODUCTION}

Rheumatoid arthritis (RA) is a disabling condition affecting approximately $0.5 \%-1 \%$ of the population in Europe and North America, ${ }^{1}$ characterised by persistent pain and stiffness of the joints, progressive joint destruction, functional disability and premature mortality. As a multifactorial disease, RA involves both the innate and the adaptive arms of the immune system. Numerous cellular and clinical studies have implicated the involvement of multiple cell types including T cells, B cells, macrophages, neutrophils depending on the

\section{Key messages}

What is already known about this subject?

- Previously, we have demonstrated a role for mitogen-activated protein kinase phosphatase 2 (MKP2) in regulating macrophage function in parasitic infections.

What does this study add?

- Here, we demonstrate a novel role for MKP-2 in regulating inflammatory arthritis through the regulation of neutrophil function.

- This study therefore further highlights a prominent role for MKP-2 in regulating immune cell function and pathological outcomes.

How might this impact on clinical practice?

- Patients presenting with arthritis could be screened for MKP-2 expression.

- Gene therapy could be used as a tool to rectify decreased or absent MKP-2 expression in patients.

immune context; yet, despite intensive study, there are still key deficits in understanding the principal mechanisms on how inflammatory arthritis develops.

A major signalling pathway implicated in the pathogenesis of RA is the MAP kinase pathway involving MAP kinases ERK, p38 and $\mathrm{JNK}^{2}{ }^{2}$ In RA, all three kinases are activated and implicated to different extents in joint inflammation, pannus formation and joint destruction. Of these three kinases, ERK has recently gained more interest, with one study demonstrating the importance of ERK in synovial cell death, where it was shown to regulate $\mathrm{TNF}-\alpha$-induced expression of B-cell activating factor through an increase in hypoxia-inducible factor- $1 \alpha .{ }^{4}$ Another study highlighted a role for ERK in TNF- $\alpha$-induced IL-8 expression in synovial fibroblasts, which contributes to synovitis. ${ }^{5}$

The kinetics and magnitude of MAP kinase activities is closely regulated by a family of dual specific phosphatases (DUSPs) known as 
the MAP kinase phosphatases. ${ }^{6}$ Three major classes exist, each with overlapping subcellular expression, kinase specificity and regulation by cellular stimulation. ${ }^{7}$ In contrast to studies involving the MAP kinases, there are very little data investigating the role of DUSPs in regulating RA. However, it has been shown that the deletion of mitogen-activated protein kinase phosphatase 1 (MKP-1) leads to enhanced severity of collagen induced arthritis (CIA) and increased cytokine release, ${ }^{8}$ while DUSP2 (PAC1) deficient mice showed a reduced response in the $\mathrm{KxB} / \mathrm{N}$ model of inflammatory arthritis. ${ }^{9}$

MKP-2 (DUSP4) is a type 1 nuclear phosphatase $\mathrm{p}^{10}$ specific for ERK and JNK in vitro. As a type 1 DUSP, MKP-2 has been viewed as a surrogate to the prototypic MKP-1 and less extensively examined. ${ }^{11}$ However, more recent studies have identified distinct roles for MKP-2 in different cellular processes and associated pathologies such as tumour development, ${ }^{12}{ }^{13}$ cancer resistance, ${ }^{14}$ cardiac myopathy, ${ }^{15}$ infection ${ }^{16}$ and inflammation. Recent studies using KO models have shown that MKP-2 protects against septic shock ${ }^{17}$ and plays a role in regulating $\mathrm{T}$ cell function. ${ }^{18}$ Moreover, MKP-2 deletion mice developed in our laboratory have been shown to be highly susceptible to parasite infection with Leishmania mexicana and Toxoplasma gondii due to alterations in macrophage iNOS/ arginase expression. ${ }^{19} 20$ Bone marrow-derived macrophages that have been stimulated with lipopolysaccharide (LPS) showed an increase in proinflammatory IL-6, IL-12, TNF $\alpha$ and a decrease in anti-inflammatory IL-10 in the absence of MKP-2 ${ }^{19}$. This cellular and functional phenotype marks MKP-2 as distinct from other MKPs and therefore this DUSP has the potential to play a unique role in the pathogenesis of other diseases.

In this study, we examined the potential role of MKP-2 in the pathogenesis of RA using MKP-2 deficient mice and the collagen antibody-induced arthritis (CAIA) model with a specific focus on neutrophil function and migration.

\section{MATERIALS AND METHODS \\ Mice}

Male MKP-2 wild-type and deficient mice were bred onto a C57BL/ 6 background as previously described ${ }^{19}$ and maintained under specific pathogen-free conditions in the animal facilities at the University of Strathclyde. Animals were used at 6-9 weeks of age and were age matched within each experiment. All experiments were conducted under Project Licenses, RP (PPL60/3439), JA (PPL60/3929) and CEL (PPL60/4297) granted by the UK Home Office and approval from the local ethical committee.

\section{Collagen antibody-induced arthritis and neutrophil depletion}

Mice received a single dose (2 $\mathrm{mg}$ ) of ArthritoMab (MD Bioscience) i.p. on day 0 , followed by a single dose (100 $\mu \mathrm{g}$ i.p.) of E. coli O55:b5 LPS (MD Bioscience) i.p. on day $3(n=5)$. Negative control mice received either phosphate buffered saline (PBS) on day 0 and LPS on day $3(n=3)$ or PBS on both days $(n=2)$ again i.p. Body weight, paw measurements and disease score were monitored daily. For paw measurements, both front and rear paw thickness were determined with a calliper and the measurements from day 0 were deducted to obtain the inflammation induced increase in paw size.

The arthritic disease score was determined as previously described: $:^{21} 0$ : no reaction, normal; 1 : mild but definite redness and swelling of the ankle or apparent redness and swelling limited to individual digits, regardless of the number of digits; 2: moderate redness and swelling of the ankle; 3: severe redness and swelling of the entire paw including digits; 4: maximally inflamed limb with involvement of multiple joints. The score was averaged for the front paws and rear paws for each mouse.

Neutrophils were depleted in vivo with a single dose of $0.25 \mathrm{mg}$ NIMP-R14 (AdipoGen) i.p. as previously described $^{22}$ and depletion monitored using flow cytometry. To control for NIMP-R14 neutrophil depletion, we used an isotype control: LEAF $^{\mathrm{TM}}$ Purified Rat IgG2b,K Isotype control Antibody (BioLegend, UK).

\section{Histology}

After termination of the experiment, the front and rear paw and knee of each mouse were removed, skinned and fixed in $10 \%$ formalin (Sigma-Aldrich). Samples were decalcified in 5.5\% EDTA for 30 days, washed and stored in $70 \%$ ethanol at $4^{\circ} \mathrm{C}$. For processing, samples were paraffin-embedded under vacuum and sections stained with H\&E at the University of Glasgow Veterinary School, before being scored blindly by a pathologist as previously described. ${ }^{23}$

\section{Flow cytometry}

Seventy microlitres of blood were taken with heparinised glass capillaries from the tail. After blocking unspecific binding with $10 \%$ mouse serum and $\mathrm{Fc}$ receptor blocking antibodies (anti-mouse CD16/32, eBioscience, UK), the cell surface was stained with conjugated antibodies for CD11b (Pe-Cy7; eBioscience, UK), and Ly-6G (IA8 clone, APC-Cy7; BioLegend) for $45 \mathrm{~min}$ at $4^{\circ} \mathrm{C}$. Red blood cells were lysed using FACS Lyse (BD Bioscience) for $10 \mathrm{~min}$ after which cells were washed and resuspended in $200 \mu \mathrm{l}$ PBS. Cells were analysed on the FACS Canto flow cytometer (BD Bioscience) using FACS Diva software.

\section{ELISA}

Mouse serum and joint homogenates were analysed individually for the presence of IL- 6 , TNF- $\alpha$, IL- $1 \beta$ and MCP-1 using the ELISA MAX Deluxe Set (BioLegend) following manufacturer's instructions. Absorbance was read at $450 \mathrm{~nm}$ using a plate reader (Spectramax 190, Molecular Devices, USA) and concentrations were determined against standard curves. 
In vivo imaging (IVIS)

Mice received $200 \mathrm{mg} / \mathrm{kg}$ luminol i.p. and were anaesthetised for imaging after $10 \mathrm{~min} .{ }^{24}$ Images were taken using the IVIS Spectrum (Caliper Life Sciences) with medium binning and 5 min exposure time after which mice were allowed to recover. Images were analysed using the Living Image software (Caliper Life Sciences). Briefly, regions of analysis of the same size were drawn using the photographs and adjusted as required using the overlay. Values were averaged from the two front and rear paws, respectively, and for each individual mouse and the background deducted. Values are expressed as photons per second $(\mathrm{p} / \mathrm{s})$.

\section{Chemotaxis assays}

Bone marrow neutrophils from MKP-2 ${ }^{+/+}$and MKP-2 ${ }^{-/-}$ mice were isolated on LS magnetic columns using the Ly-6G positive selection kit (Miltenyi Biotec GmbH, Germany) following manufacturer's instructions. Chemotaxis was assessed using 24-well transwell plates containing polycarbonate filter membranes of $10 \mu \mathrm{m}$ thickness and $3 \mu \mathrm{m}$ pore size (Corning). Neutrophils were resuspended in complete Roswell Park Memorial Institute (RPMI) media at $2 \times 10^{5} / 100 \mu \mathrm{l}$ and were added to the upper chamber. The lower chamber was filled with $600 \mu \mathrm{l}$ complete RPMI supplemented with either nothing (negative control), LPS $(0.5 \mu \mathrm{g} / \mathrm{mL})$, C5a $(20 \mathrm{nM})$ or $\mathrm{LTB}_{4}(300 \mathrm{nM})$. Each variable was carried out in triplicate. Following 3 hour incubation at $37^{\circ} \mathrm{C}$, the contents of the lower chamber were collected and counted. Results were expressed as percentage migration ( (number of migrated cells/total number of cells) $\times 100)$.

\section{Western blotting}

Neutrophils were isolated as described above and re-suspended in complete RPMI, $1 \times 10^{6}$ cells were used per treatment point. Following stimulation samples were analysed by Western blotting as outlined previously. ${ }^{19}$

\section{Statistics}

Statistical analysis was performed using GraphPad Prism (V.4.0, San Diego, California, USA). All error bars are shown as SE of the mean (SEM), with two-tailed unpaired t-test, with the exception of Western blots, which were one-way ANOVA with Bonferroni's post-test $(* \mathrm{p}<0.05, * * \mathrm{p}<0.01, * * * \mathrm{p}<0.001)$.

\section{RESULTS}

\section{MKP-2 deficiency results in increased inflammation and disease pathology}

MKP-2 deficient mice showed a significant increased disease score compared with wild type mice for both front and rear paws. In contrast, control mice showed no signs of pathology (figure 1A). Consistent with an increased disease score, these mice showed a significant increase in paw size (online supplementary figure S1). Analysis of the serum at day 9 showed a dramatic increase of cytokines IL- 6 and TNF- $\alpha$ as well as chemokine MCP-1 in MKP-2 ${ }^{-/}$mice compared with wild type and control mice (figure 1B). We further determined the presence of these cytokines in joint homogenates. However, while slightly increased in MKP-2 ${ }^{-/}$, these results are not statistically significant (online supplementary figure S2).

Histology on H\&E stained sections of front paws, rear paws and knees showed a significant joint pathology in $\mathrm{MKP}-2^{-/-}$mice with increased infiltration and hyperplasia (figure 1C). Taken together, these results indicate that MKP-2 deficiency results in significant disease pathology in collagen antibody-induced arthritis.

\section{Inflammation is linked to increased neutrophil migration into} the joints and can be influenced by neutrophil depletion Neutrophils have been previously linked to arthritic disease. ${ }^{25}$ In order to visualise myeloperoxidase-containing neutrophil influx into the joints, mice were injected with luminol. In vivo imaging revealed that MKP-2 deficient mice showed a significant increase in neutrophils in the joints compared with wild type and control mice (figure 2).

We next investigated how depletion of neutrophils affects disease development. Mice were divided into four groups; an overview of the respective treatments is shown in figure 3A. Mice in the positive control group (+ve control) received ArthritoMab at day 0, followed by LPS at day 3 and in addition received an isotype control instead of the neutrophil depletion antibody. These mice developed pathology with $\mathrm{MKP}^{-} 2^{-/-}$showing higher disease scores similar to previous observations. Mice of the negative control group (-ve control) were injected with PBS on both days but in addition received neutrophil depleting antibody NIMP-R14 6 hour prior to the second PBS injection to ensure that neutrophil depletion on its own does not influence disease.

The successful depletion of neutrophils was monitored by flow cytometry and by in vivo imaging (figure 3B) and as expected, these mice did not show any significant pathology. Mice of the prophylactic group were treated with NIMP-R14 6 hours prior to LPS injection and before the onset of disease. No disease pathology in respect to arthritis was found in these mice. Similar effects have been found in the therapeutic group, where neutrophil depletion at day 6 , after onset of disease, resulted in rapid amelioration of disease symptoms (figure 3C).

Concentrations of the cytokines TNF- $\alpha$ and IL- 6 were below detection (TNF- $\alpha$ ) or down to levels of the negative controls (figure 3D and online supplementary figure 4). Interestingly, concentrations of MCP-1 were unaffected by neutrophil depletion, thus suggesting that neutrophils are not the source of this chemokine.

At this point, most animals in the prophylactic group treated with the NIMP-R14 antibody prior to LPS became severely ill and showed signs of endotoxic shock. Although mice in this group made a full recovery, 
A

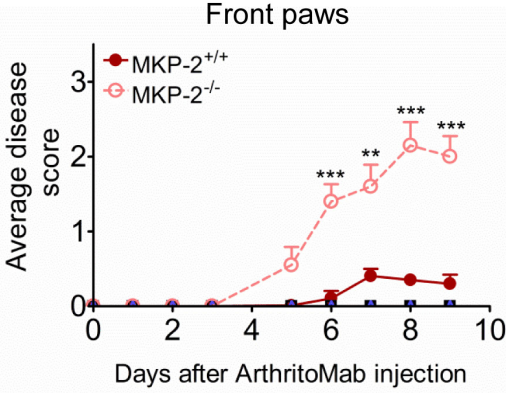

Rear paws

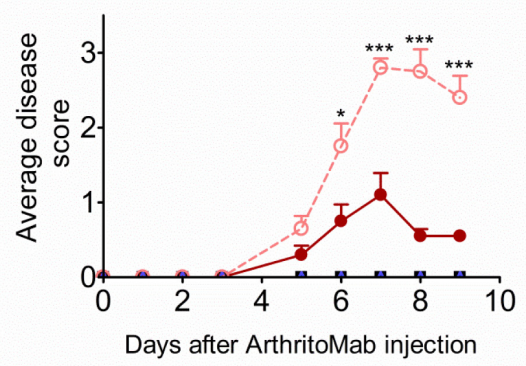

B

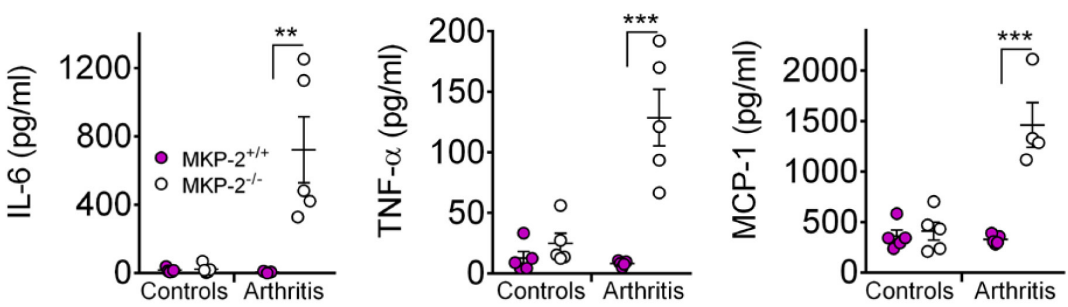

C

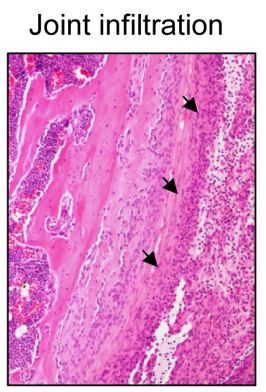

Joint infiltration

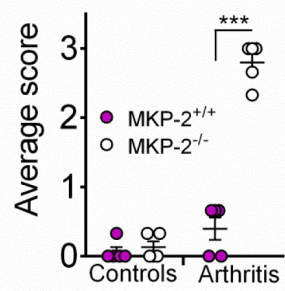

Hyperplasia

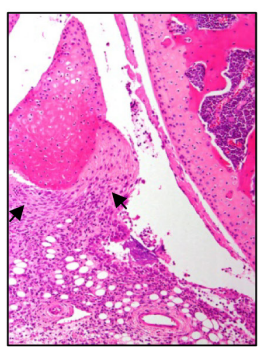

Figure 1 MKP-2 deficiency results in increased inflammation and arthritis related pathology. Mice received a single dose of ArthritoMab at day 0 and a single injection of LPS at day 3. Negative control groups received either PBS on both days or PBS on day 0 followed by a single dose of LPS at day 3. Disease development was observed over a period of 9 days. (A) Arthritic score was determined as described in materials and methods, MKP $-2^{+/+}$(solid line and symbols), MKP-2 $2^{-/-}$(dashed line and open symbols). (B) Serum cytokines were determined by ELISA, controls are a combination of all negative control groups. (C) H\&E stained tissue sections (left panels) of front paw, rear paw and knee joints were scored blindly and evaluated for infiltration and hyperplasia (right panels). Arrows point to the respective pathology shown on a representative example of an MKP $-2^{-/-}$knee joint. Scores of all three joints were averaged per mouse and plotted as MKP- $2^{+/+}$(solid bars) and MKP-2 ${ }^{-}$ 1- (open bars). For the total score, scores for joint infiltration and hyperplasia were summed for each individual mouse. All error bars are shown as SE of the mean (SEM). ${ }^{*} \mathrm{P} \leq 0.05$, ${ }^{\star \star} \mathrm{P} \leq 0.01$, ${ }^{\star \star \star} \mathrm{P} \leq 0.001,{ }^{* \star \star \star} \mathrm{P}<0.0001$, two-tailed unpaired $\mathrm{T}$ test. Data shown are representative of three independent experiments (mouse numbers; pilot-PBS ctrl=2, LPS ctrl=2, ArthritoMab/LPS=6; Exp 2-PBS ctrl=4, LPS ctrl=6, ArthritoMab/LPS=10; Exp 3-PBS ctrl=4, LPS ctrl=6, ArthritoMab/LPS=10 *(Totals across MKP ${ }^{+/+}$ and $\mathrm{MKP}^{-/}$genotypes, with equal numbers in each).

the serum across the groups showed a high variation of IL-6, MCP-1 and TNF- $\alpha$ (online supplementary figure S4); therefore, no conclusion can be drawn as these are more likely to be the result of a cytokine storm than arthritis. For this reason also, this experiment was only conducted twice, and for the second experiment, the prophylactic group was omitted due to the previous endotoxic shock observed.

Histological analysis of affected joints showed that treatment with neutrophil depleting antibody abolished arthritis related pathology in $\mathrm{MKP}^{-} 2^{-/-}$mice (online supplementary figure S3) to the level of naive mice. 

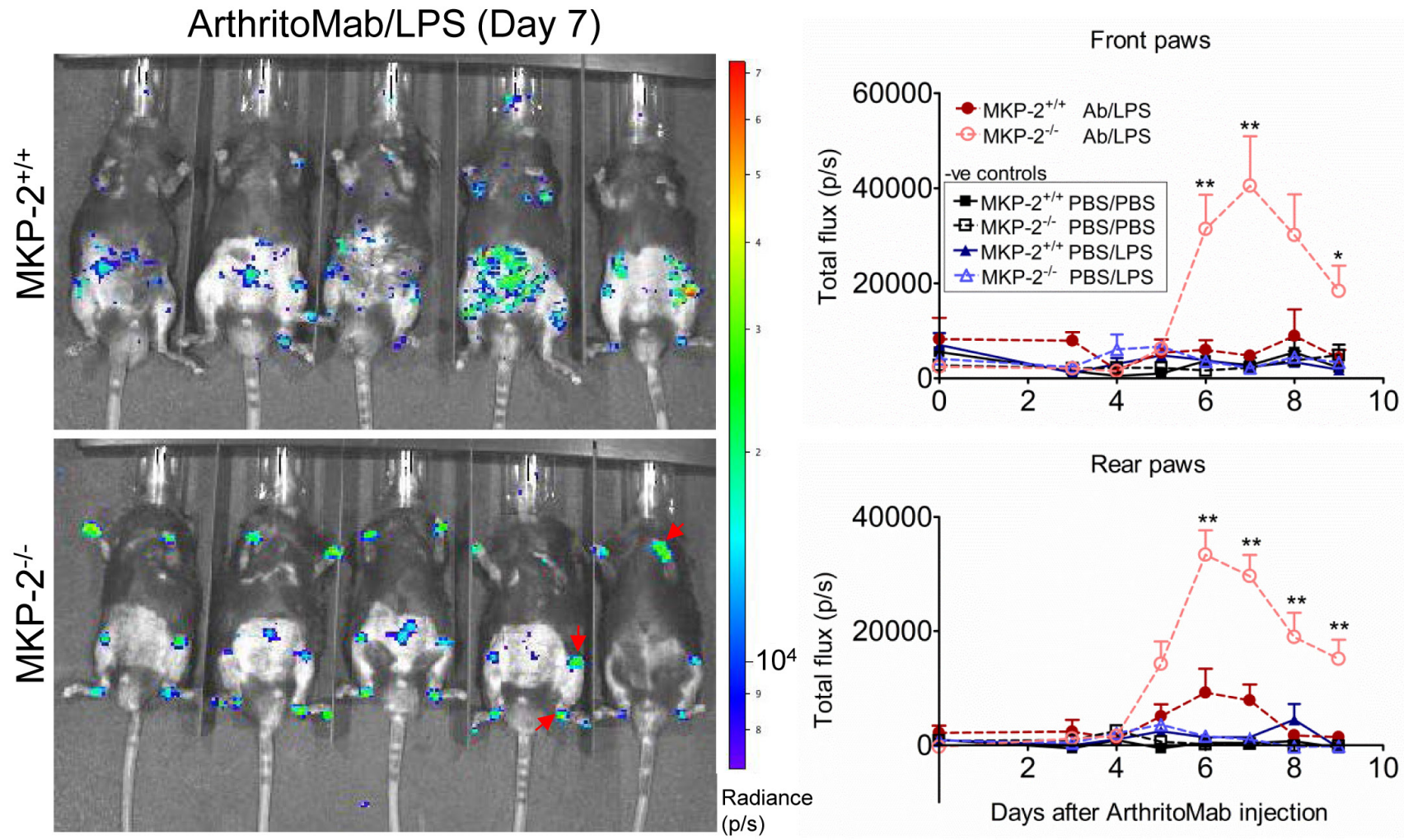

Figure $2 \mathrm{MKP}-2^{-/-}$mice show an increased neutrophil infiltration into the joints. Mice received a single dose of ArthritoMab $(\mathrm{AB})$ at day 0 and a single injection of LPS at day 3. Negative control groups received either PBS on both days or PBS on day 0 followed by a single dose of LPS at day 3. Disease development was observed over a period of 9 days. Neutrophils were visualised 10 min after i.p. injection of luminol and resulting bioluminescence was detected using in vivo imaging. Shown is a representative image from day 7 (left panels) and the quantification over the duration of the experiment for front and rear paws (right). Red arrows indicate neutrophil influx. Values are expressed as photons per second (P/S). All error bars are shown as $\mathrm{SE}$ of the mean (SEM). ${ }^{*} \mathrm{P} \leq 0.05,{ }^{\star \star} \mathrm{P} \leq 0.01$, two-tailed unpaired $\mathrm{T}$ test. Data shown are representative of three independent experiments (mouse numbers; pilot-PBS ctrl=2, LPS ctrl=2, ArthritoMab/LPS=6; Exp 2-PBS ctrl=4, LPS ctrl=6, ArthritoMab/ LPS=10; Exp 3-PBS ctrl=4, LPS ctrl=6, ArthritoMab/LPS $=10$ *(Totals across $\mathrm{MKP}^{+/+}$and $\mathrm{MKP}^{-/-}$genotypes, with equal numbers in each).

Overall, these experiments point to neutrophils as the main mediator of inflammation since specific depletion of this cell type prevents or ameliorates disease and cytokine levels are ablated after neutrophil depletion.

\section{MKP-2 deficient neutrophils show increased chemotaxis}

We next performed transwell assays to determine if the increased migration is a result of MKP-2 deficiency. As chemo-attractants we chose C5a and Leukotriene B4 $\left(\mathrm{LTB}_{4}\right)$ as these agents are known to have strong chemotactic effects on neutrophils. Neutrophils showed an increased migration towards both $\mathrm{C} 5 \mathrm{a}$ and $\mathrm{LTB}_{4}$. The migratory effect was significantly enhanced in $\mathrm{MKP}^{-2^{-1}}$ neutrophils in response to $\mathrm{LTB}_{4}(\mathrm{p}<0.05)$, but no significant difference was seen with C5a (figure $4 \mathrm{~A}$ ).

We then sought to correlate this difference with changes in MAP kinase activity. We found that LTB $_{4}$ strongly phosphorylated ERK in both groups; however, there was no significant difference between MKP-2 ${ }^{-/}$and MKP- $2^{+/+}$neutrophils (figure $4 \mathrm{~B}$ ). LTB $_{4}$ did not appear to activate the p38 pathway and therefore no comparisons could be made. In comparison we found that in

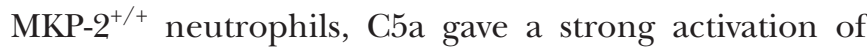
ERK peaking at $5 \mathrm{~min}$ and this response was markedly reduced in MKP-2/- neutrophils $(\mathrm{p}<0.001)$ (figure $4 \mathrm{C}$ ). C5a was also able to phosphorylate p38 MAPK, although to a smaller level (n.s), and this was not significantly altered by DUSP4 deletion (figure 4C). In contrast, no JNK phosphorylation was detected in any of the samples (not shown).

\section{DISCUSSION}

Using MKP-2 deficient mice developed in our laboratory, we present for the first time evidence that MKP-2 plays a key protective role in inflammatory arthritis. MKP-2 has been implicated in both inflammatory responses and infection with particular emphasis on macrophages and T-cell function. In this study, we show that in an innate model of arthritis neutrophils are also influenced by MKP-2, and MKP-2 deficient neutrophils migrate to the joint more rapidly to mediate join destruction through enhanced cytokine release. 
A

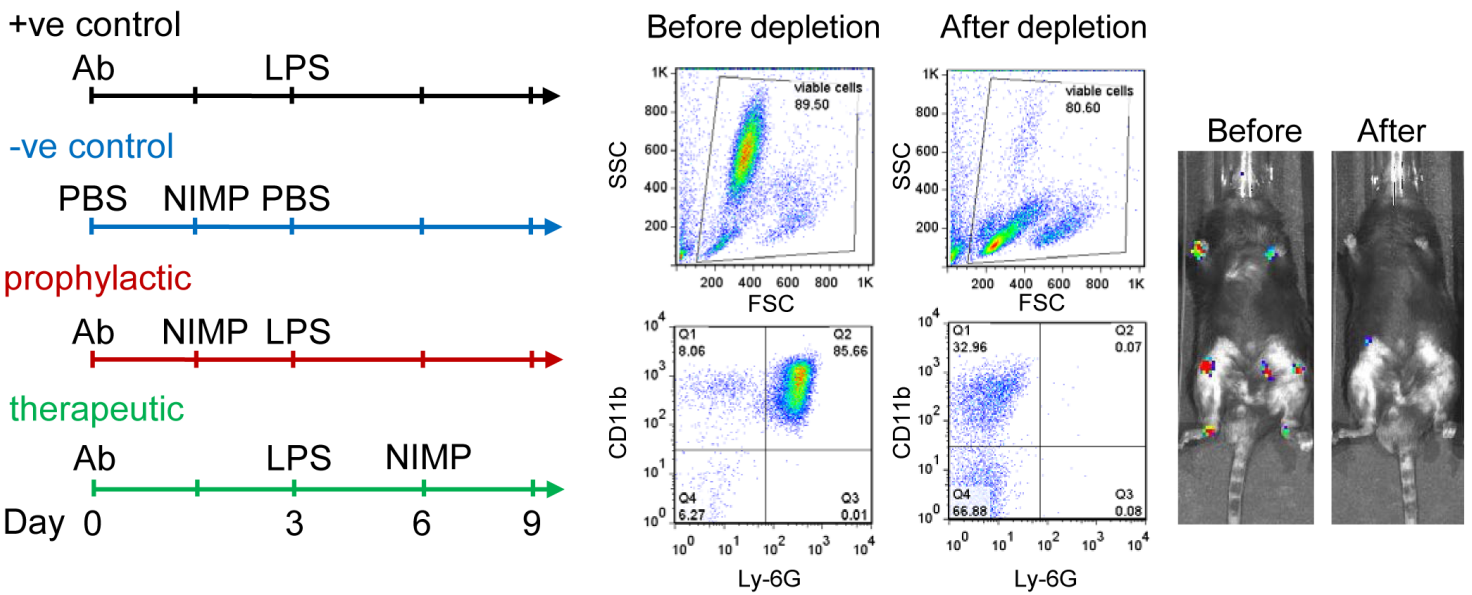

C

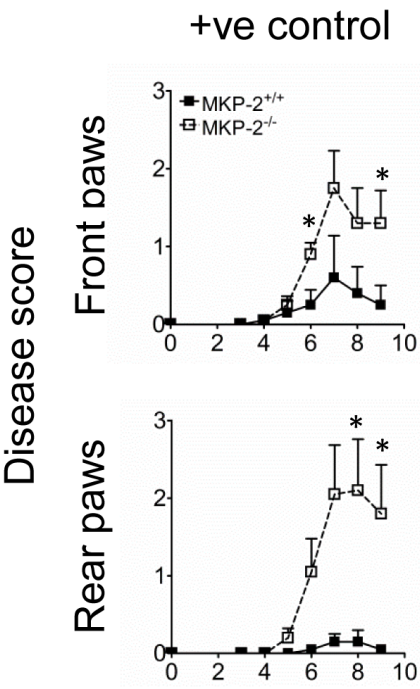

-ve control
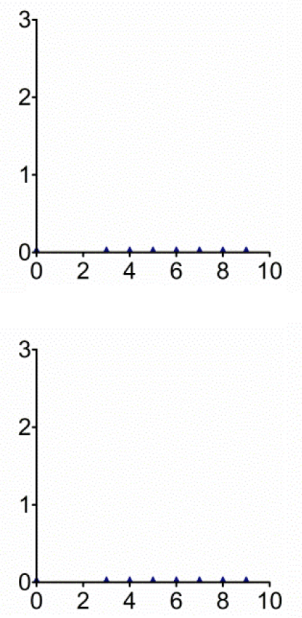

prophylactic
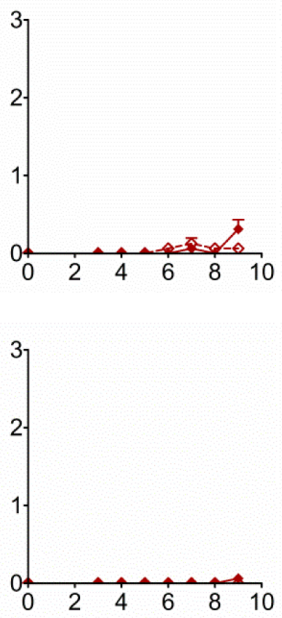

therapeutic
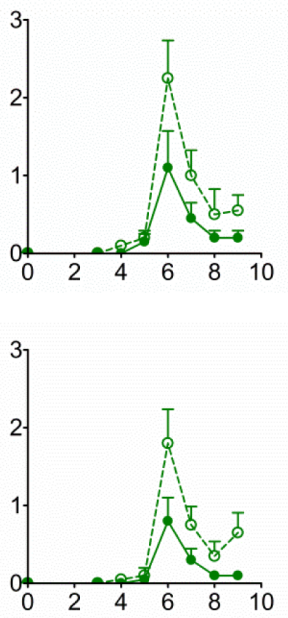

Days after ArthritoMab injection

D

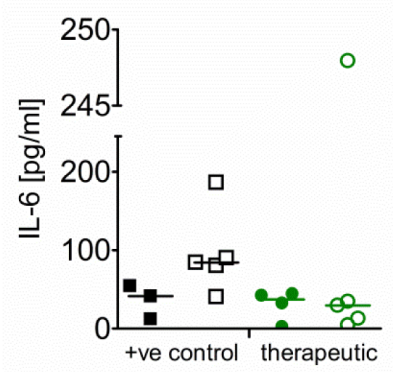

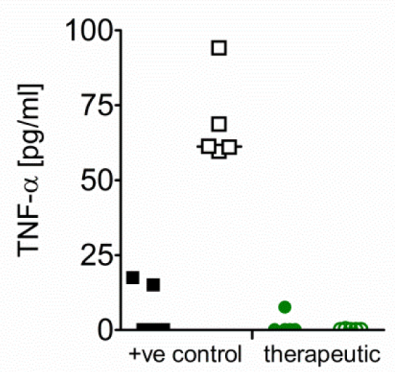

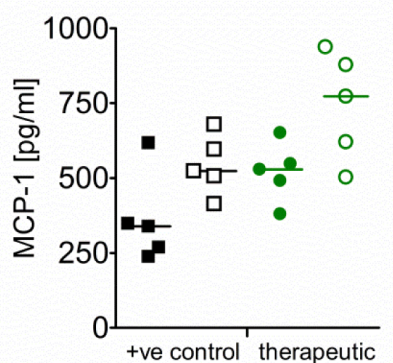

Figure 3 Neutrophil depletion prevents and ameliorates inflammation. Mice were divided into four groups of five animals per genotype and received treatment as shown in (A). All groups but the negative controls received ArthritoMab at day 0 and LPS at day 3. Neutrophils at day 3 were depleted 6 hour prior to i.p. injection of LPS or PBS in the prophylactic and negative control groups, respectively. The therapeutic groups received NIMP after onset of disease at day 6. (B) Neutrophil depletion from blood before and after treatment with NIMP from MKP-2/- mice from the therapeutic group was monitored using flow cytometry with specific staining for CD11b and Ly-6G (left panels) and also via bioluminescence (right panels) following luminol injection. (C) Disease scores were determined as described in materials and methods, closed symbols depict MKP-2 $2^{+/+}$mice and open symbol depicts $\mathrm{MKP}^{-/-}$mice. (D) Serum cytokines were determined using ELISA. All error bars are shown as SE of the mean (SEM). Data shown are representative of two independent experiments. (Mouse numbers; Exp 1: ArthritoMab/LPS=10, PBS/ $\mathrm{NIMP}=10$, Prophylactic=10 and Therapeutic=10; Exp 2: ArthritoMab/LPS=6, PBS/NIMP=0, Prophylactic=0 and Therapeutic=6; *(Totals across $\mathrm{MKP}^{+/+}$and $\mathrm{MKP}^{-/-}$genotypes, with equal numbers in each). 


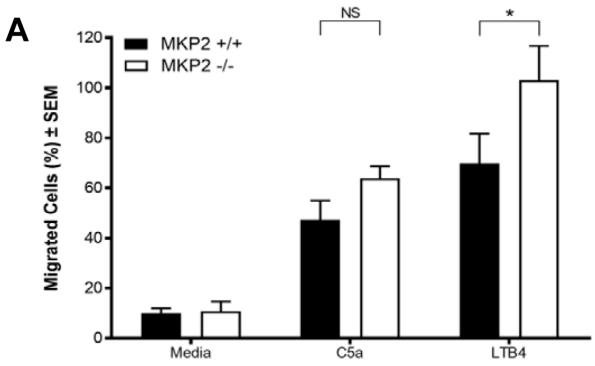

B LTB4 (300 nM)
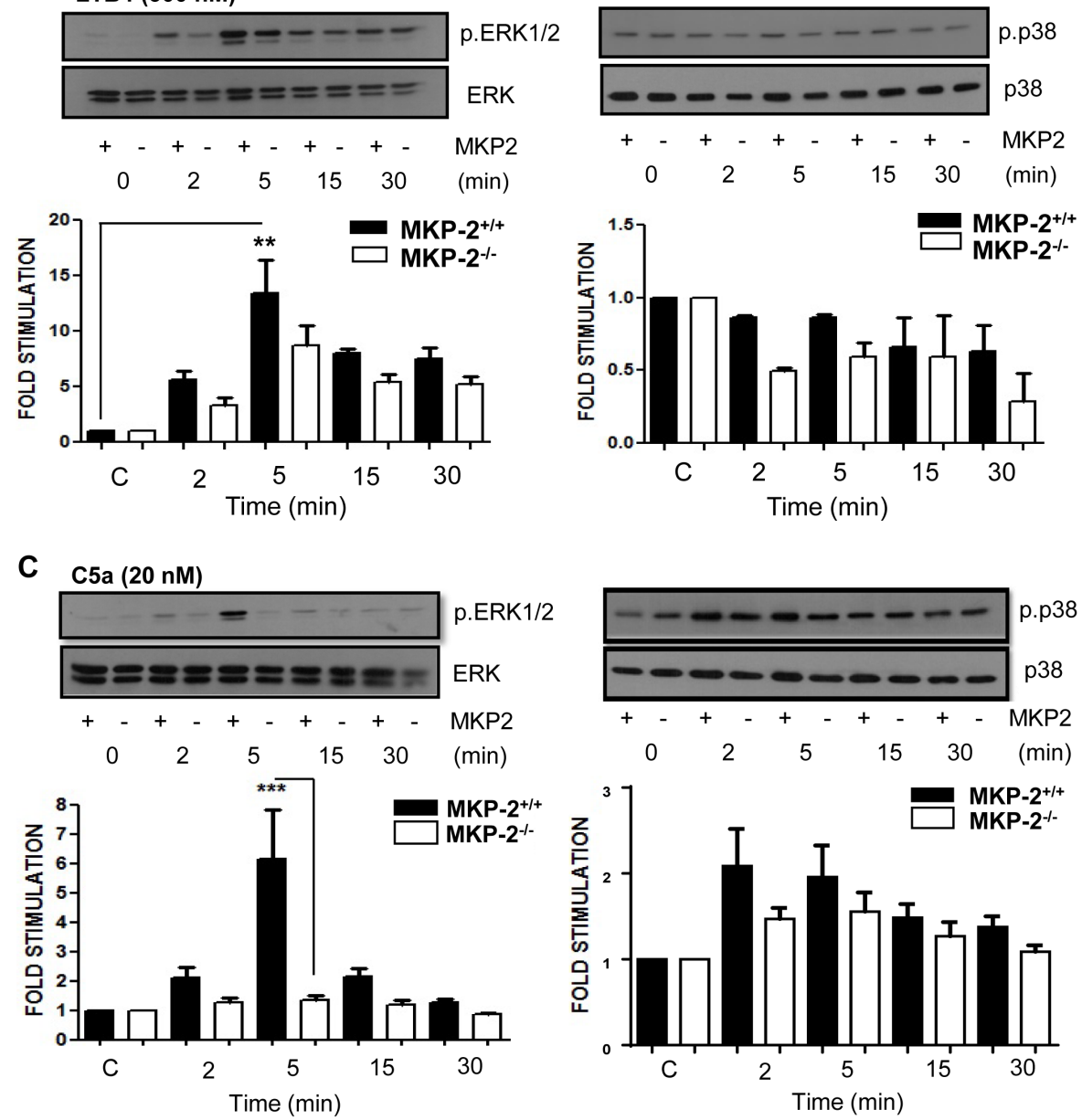

Figure 4 MKP-2 deficient neutrophils show increased chemotactic activity in vitro. (A) Purified neutrophils were subjected to Transwell migration assays using LPS $(0.5 \mu \mathrm{g} / \mathrm{mL})$, C5a $(20 \mathrm{nM})$ and LTB $(300 \mathrm{nM})$ as chemoattractants. Migrating cells are shown as percentage of the overall population. (B) Neutrophils were stimulated with $\mathrm{LTB}_{4}(300 \mathrm{nM})$ over a time course and cell extracts were analysed for the presence of phosphorylated ERK and p38. (C) Neutrophils were stimulated with C5a (20 nM) over a time course and cells' extracts analysed for the presence of phosphorylated ERK and p38. Membranes were stripped and reprobed for their respective totals. All error bars are shown as SE of the mean (SEM), ${ }^{*} p \leq 0.05,{ }^{* *} p \leq 0.01,{ }^{* \star *} p \leq 0.001$. $(A)$ Two-tailed unpaired t test, $(B, C)$ One-way ANOVA with Bonferroni's post-test. (C,D) Closed symbols depict MKP-2 ${ }^{+/+}$mice and open symbol depict $\mathrm{MKP}^{-/-}$mice. Data shown are representative of three independent experiments (mouse numbers - four mice from each genotype, so a total of eight were used per each independent experiment).

In this study, enhanced damage to the joint in MKP-2mice was accompanied by a marked increase in serum levels of MCP-1, IL-6 and TNF $\alpha$. This is in line with observations made in $\mathrm{MKP}-1^{-/}$mice which received LPS injections ${ }^{8}$ and suggests common regulatory functions between nuclear inducible DUSPs. However, these results are different from another DUSP-4 deletion model which showed reduced serum levels of TNF $\alpha$ in response to LPS. ${ }^{17}$ Differences in the dose of LPS and the time at which TNF $\alpha$ was measured is likely to explain the differences; however, in our hands, MKP- $2^{--}$macrophages also show enhanced TNF $\alpha$ production, ${ }^{19}$ suggesting an intrinsic difference in cytokine production in DUSP-4 deficient cells of myeloid origin compared with WT.

Nevertheless, to our surprise, depletion of neutrophils completely abrogated serum TNF $\alpha$, which indicates that neutrophils are the main producer of this cytokine in the CAIA model. This is consistent with other studies 
indicating the potential of neutrophils to be a source of $\mathrm{TNF} \alpha$ and other related ligands following innate immune challenge. ${ }^{26}$ Interestingly, MCP-1, which is associated with inflammatory arthritis, was not affected suggesting a different source. While many cells are able to produce MCP-1, it has been shown that monocyte/ macrophages are the main producer. ${ }^{27}$ When examining joint pathology, we found that neutrophil depleted mice showed a significantly reduced pathology score. This raises the question as to what extent each cytokine/ chemokine contributes to joint destruction. It is known that proinflammatory cytokines, TNF $\alpha$, IL-6 and IL-1 can indirectly trigger osteoclastogenesis by upregulating RANKL on the surface of osteoblasts. ${ }^{28}$ On the other hand, MCP-1 induces RANKL expression on osteoclast precursor cells, a process which is ERK dependent. ${ }^{29}$ It is therefore possible that the lack of TNF $\alpha$ caused after neutrophil depletion results in a decrease of RANKL on osteoblasts, crucial for osteoclast activation, which would explain why the presence of MCP-1 alone did not result in joint damage. This would be something that could be investigated in future studies.

Since neutrophils appear to be the key cell type responsible for the pathology observed, we addressed the question whether MKP-2 deficiency alters chemotactic activity. Isolated neutrophils displayed migration towards both C5a and $\mathrm{LTB}_{4}$; however, MKP-2/- neutrophils showed a significantly increased migration towards $\mathrm{LTB}_{4}$, a potent chemoattractant to neutrophils, and indeed Finsterbusch and coworkers have shown that neutrophils recruited by these chemoattractants release TNF $\alpha$ leading to microvascular leakage and inflammation. ${ }^{30}$ Recent studies have revealed that $\mathrm{LTB}_{4}$ is a key mediator in a complement, lipid, cytokine and chemokine cascade that first initiates and then sustains neutrophilic inflammation in inflammatory arthritis ${ }^{31}$

Stimulating neutrophils with either $\mathrm{LTB}_{4}$ or C5a resulted in a strong phosphorylation of ERK which was reduced following MKP-2 deletion. Work by Liu et al showed that ERK is a negative regulator of migration ${ }^{32}$ and this would be consistent with our finding that in MKP- $2^{-/}$neutrophils ERK is reduced, negative regulation is abrogated and migration enhanced. In contrast, p38 MAPK, believed to provide a positive chemotaxis signal, was not significantly altered in our studies. However, it should be noted that our expectation from a signalling perspective is that ERK activation may have been enhanced due to lack of regulatory dephosphorylation by MKP-2. This suggests MKP-2 deletion may have effects which regulate other components of the ERK cascade including receptor expression and functional coupling to upstream intermediates. A number of studies including our own have also suggested that MKP-2 can regulate cellular function in a way which is not dependent on the normal predicted effects on ERK and JNK. ${ }^{1133}$ Indeed, recently MKP-2 has been shown to interact and regulate the cell cycle protein VRK-1 independently of phosphatase activity suggesting multiple cellular effects. ${ }^{35}$ Another coincidental explanation may be pathway redundancy and/or cross talk with MKP-1, such that ERK signalling is altered in a different way. ${ }^{17}$

Finally, another possibility, not examined in this study, is that MKP-2 may regulate apoptosis and indeed MKP-2 deficient neutrophils are present in far greater numbers and appear to reside in the joint for longer, possibly prolonging the release of cytokines. This would be consistent with studies that show delayed neutrophil apoptosis in response to $\mathrm{LTB}_{4}^{36}$ and L. major infection. ${ }^{37}$ Indeed, MKP-2 deletion results in the inhibition of B- cell apoptosis $^{38}$ and also enhances $\mathrm{T}$ cell proliferation ${ }^{18}$ consistent with this idea.

Overall, our novel findings suggest that MKP-2 has the potential to play a major role in regulating RA due to a direct effect on neutrophil function, with the potential to effect macrophages, osteoclast formation and bone development. The use of specific conditional knockout mice would allow further dissection of these events. However, it remains that MKP-2 may be an attractive target for gene replacement therapy.

Contributors All authors contributed to this manuscript, with experimental design, execution of experiments, data analysis and manuscript preparation.

Funding This study was funded by Wellcome Trust (WT089273MA).

Competing interests None declared.

Patient consent Not required.

Ethics approval University of Strathclyde, home office approval and local ethical committee.

Provenance and peer review Not commissioned; externally peer reviewed.

Data sharing statement All data generated during this study have been included either in the main manuscript or as supplemental figures.

Open access This is an open access article distributed in accordance with the Creative Commons Attribution 4.0 Unported (CC BY 4.0) license, which permits others to copy, redistribute, remix, transform and build upon this work for any purpose, provided the original work is properly cited, a link to the licence is given and indication of whether changes were made. See: http://creativecommons.org/ licenses $/$ by $/ 4.0$

\section{REFERENCES}

1. Alamanos $\mathrm{Y}$, Drosos AA. Epidemiology of adult rheumatoid arthritis. Autoimmun Rev 2005;4:130-6.

2. Lindstrom TM, Robinson WH. A multitude of kinases--which are the best targets in treating rheumatoid arthritis? Rheum Dis Clin North Am 2010;36:367-83.

3. Schett G, Tohidast-Akrad M, Smolen JS, et al. Activation, differential localization, and regulation of the stress-activated protein kinases, extracellular signal-regulated kinase, c-JUN N-terminal kinase, and p38 mitogen-activated protein kinase, in synovial tissue and cells in rheumatoid arthritis. Arthritis Rheum 2000;43:2501-12.

4. Lee JW, Lee J, Um SH, et al. Synovial cell death is regulated by TNF- $\alpha$-induced expression of B-cell activating factor through an ERK-dependent increase in hypoxia-inducible factor-1 $\alpha$. Cell Death Dis 2017;8:e2727.

5. Namba S, Nakano R, Kitanaka T, et al. ERK2 and JNK1 contribute to TNF- $\alpha$-induced IL- 8 expression in synovial fibroblasts. PLoS One 2017;12:e0182923.

6. Keyse SM. Dual-specificity MAP kinase phosphatases (MKPs) and cancer. Cancer Metastasis Rev 2008;27:253-61.

7. Caunt CJ, Keyse SM. Dual-specificity MAP kinase phosphatases (MKPs): shaping the outcome of MAP kinase signalling. Febs $J$ 2013;280:489-504.

8. Salojin KV, Owusu IB, Millerchip KA, et al. Essential role of MAPK phosphatase-1 in the negative control of innate immune responses. J Immunol 2006;176:1899-907.

9. Jeffrey KL, Brummer T, Rolph MS, et al. Positive regulation of immune cell function and inflammatory responses by phosphatase PAC-1. Nat Immunol 2006;7:274-83. 
10. Guan KL, Butch E. Isolation and characterization of a novel dual specific phosphatase, $\mathrm{HVH} 2$, which selectively dephosphorylates the mitogen-activated protein kinase. J Biol Chem 1995;270:7197-203.

11. Lawan A, Torrance E, Al-Harthi S, et al. MKP-2: out of the DUSP-bin and back into the limelight. Biochem Soc Trans 2012;40:235-9.

12. Chitale D, Gong Y, Taylor BS, et al. An integrated genomic analysis of lung cancer reveals loss of DUSP4 in EGFR-mutant tumors. Oncogene 2009;28:2773-83.

13. Waha A, Felsberg J, Hartmann W, et al. Epigenetic downregulation of mitogen-activated protein kinase phosphatase MKP-2 relieves its growth suppressive activity in glioma cells. Cancer Res 2010:70:1689-99.

14. Balko JM, Cook RS, Vaught DB, et al. Profiling of residual breast cancers after neoadjuvant chemotherapy identifies DUSP4 deficiency as a mechanism of drug resistance. Nat Med 2012;18:1052-9.

15. Auger-Messier M, Accornero F, Goonasekera SA, et al. Unrestrained p38 MAPK activation in Dusp1/4 double-null mice induces cardiomyopathy. Circ Res 2013;112:48-56.

16. Schroeder J, McGachy HA, Woods S, et al. T Cell Hyporesponsiveness against leishmania major in map kinase phosphatase (mkp) 2 deficient c57bl/6 mice does not alter the healer disease phenotype. PLoS Negl Trop Dis 2013;7;7:e2064.

17. Cornell TT, Rodenhouse $\mathrm{P}, \mathrm{Cai} \mathrm{Q}$, et al. Mitogen-activated protein kinase phosphatase 2 regulates the inflammatory response in sepsis. Infect Immun 2010;78:2868-76.

18. Huang $\mathrm{C}-\mathrm{Y}$, Lin $\mathrm{Y}-\mathrm{C}, \mathrm{Hsiao} \mathrm{W}-\mathrm{Y}$, et al. DUSP4 deficiency enhances CD25 expression and CD4 ${ }^{+} \mathrm{T}$-cell proliferation without impeding T-cell development. Eur J Immunol 2012;42:476-88.

19. Al-Mutairi MS, Cadalbert LC, McGachy HA, et al. MAP kinase phosphatase- 2 plays a critical role in response to infection by Leishmania mexicana. PLoS Pathog 2010;6:e1001192.

20. Woods S, Schroeder J, McGachy HA, et al. MAP kinase phosphatase-2 plays a key role in the control of infection with Toxoplasma gondii by modulating iNOS and arginase- 1 activities in mice. PLoS Pathog 2013;9:e1003535.

21. Courtenay JS, Dallman MJ, Dayan AD, et al. Immunisation against heterologous type II collagen induces arthritis in mice. Nature 1980;283:666-8.

22. McFarlane E, Perez C, Charmoy M, et al. Neutrophils contribute to development of a protective immune response during onset of infection with Leishmania donovani. Infect Immun 2008;76:532-41.

23. lerna M, Kerr A, Scales $\mathrm{H}$, et al. Supplementation of diet with krill oil protects against experimental rheumatoid arthritis. BMC Musculoskelet Disord 2010;11:136.
24. Gross S, Gammon ST, Moss BL, et al. Bioluminescence imaging of myeloperoxidase activity in vivo. Nat Med 2009;15:455-61.

25. Tanaka D, Kagari T, Doi H, et al. Essential role of neutrophils in antitype II collagen antibody and lipopolysaccharide-induced arthritis. Immunology 2006;119:195-202.

26. Mantovani A, Cassatella MA, Costantini C, et al. Neutrophils in the activation and regulation of innate and adaptive immunity. Nat Rev Immunol 2011;11:519-31.

27. Deshmane SL, Kremlev S, Amini S, et al. Monocyte chemoattractant protein-1 (MCP-1): an overview. J Interferon Cytokine Res 2009;29:313-26.

28. Souza PP, Lerner UH. The role of cytokines in inflammatory bone loss. Immunol Invest 2013:42:555-622.

29. Binder NB, Niederreiter B, Hoffmann O, et al. Estrogen-dependent and $\mathrm{C}-\mathrm{C}$ chemokine receptor-2-dependent pathways determine osteoclast behavior in osteoporosis. Nat Med 2009;15:417-24.

30. Finsterbusch M, Voisin M-B, Beyrau M, et al. Neutrophils recruited by chemoattractants in vivo induce microvascular plasma protein leakage through secretion of TNF. J Exp Med 2014;211:1307-14

31. Miyabe Y, Miyabe C, Luster AD. LTB and BLT1 in inflammatory arthritis. Semin Immunol 2017;33:52-7.

32. Liu X, Ma B, Malik AB, et al. Bidirectional regulation of neutrophil migration by mitogen-activated protein kinases. Nat Immunol 2012;13:457-64.

33. Lawan A, Al-Harthi S, Cadalbert L, et al. Deletion of the dual specific phosphatase-4 (dusp-4) gene reveals an essential non-redundant role for map kinase phosphatase-2 (mkp-2) in proliferation and cell survival. J Biol Chem 2011;286:12933-43.

34. Hasegawa T, Enomoto A, Kato T, et al. Roles of induced expression of MAPK phosphatase-2 in tumor development in RET-MEN2A transgenic mice. Oncogene 2008;27:5684-95.

35. Jeong MW, Kang TH, Kim W, et al. Mitogen-activated protein kinase phosphatase 2 regulates histone $\mathrm{H} 3$ phosphorylation via interaction with vaccinia-related kinase 1. Mol Biol Cell 2013;24:373-84.

36. Pétrin D, Turcotte S, Gilbert AK, et al. The anti-apoptotic effect of leukotriene B4 in neutrophils: a role for phosphatidylinositol 3-kinase, extracellular signal-regulated kinase and Mcl-1. Cell Signal 2006;18:479-87.

37. Sarkar A, Aga E, Bussmeyer U, et al. Infection of neutrophil granulocytes with Leishmania major activates ERK $1 / 2$ and modulates multiple apoptotic pathways to inhibit apoptosis. Med Microbiol Immunol 2013;202:25-35.

38. Ramesh S, Qi XJ, Wildey GM, et al. TGF beta-mediated BIM expression and apoptosis are regulated through SMAD3-dependent expression of the MAPK phosphatase MKP2. EMBO Rep 2008;9:990-7. 